\title{
Irinotecan-platinum combination therapy for previously untreated extensive-stage small cell lung cancer patients: a meta- analysis
}

Fei Xu ${ }^{1,2}$, Xiaoli Ren ${ }^{1}$, Yuan Chen ${ }^{1}$, Qianxia $\mathrm{Li}^{1}$, Ruichao $\mathrm{Li}^{1}, \mathrm{Yu}$ Chen ${ }^{1}$ and Shu Xia ${ }^{1 *}$

\begin{abstract}
Background: There is still a debate regarding whether regimens combining irinotecan with platinum could replace regimens combining etoposide with platinum, as first-line chemotherapy for extensive-stage small cell lung cancer (ES-SCLC). We performed a meta-analysis to compare these regimens as first-line chemotherapy for ES-SCLC.

Methods: A literature search for randomized controlled trials was performed using the Cochrane Library, PubMed, and Embase. The inverse variance method was used to estimate summary hazard ratios and their 95\% confidence intervals for overall survival and progression free survival. Relative risk was used to estimate the overall response rate, disease control rate, 1-year survival, 2-year survival, and adverse event data.

Result: Nine randomized controlled trials (2451 patients) were included. Regimens combining irinotecan and platinum improved overall survival, progression-free survival and overall response rate compared to combination etoposide and platinum regimens. Meanwhile, superior progression-free survival and overall response rate outcomes were observed in the Asian subgroup of patients. These patients receiving a combination irinotecan and platinum regimen experienced grade 3-4 diarrhea more frequently and experienced less hematologic toxic events than the non-Asian groups.

Conclusions: Our data suggest that a combination irinotecan and platinum regimen can prolong overall survival, progression-free survival and overall response rate for patients with ES-SCLC as compared to a combination etoposide and platinum regimen. And the Asian patients could benefit from irinotecan combined with platinum easier.
\end{abstract}

Keywords: Small cell lung cancer, Extensive-staged, Irinotecan, Etoposide, Meta-analysis

\section{Background}

Lung cancer, which represents $13 \%$ of newly diagnosed cancers worldwide, is the most common tumor type [1]. Small cell lung cancer (SCLC) accounts for approximately $15 \%$ of new cases of annually diagnosed lung cancer, and up to $25 \%$ of lung cancer deaths each year [2]. Approximately two-thirds of patients with SCLC are

\footnotetext{
* Correspondence: xiashutj@hotmail.com

'Department of Oncology, Tongji Hospital, Tongji Medical College of Huazhong University of Science and Technology, Wuhan, People's Republic of China

Full list of author information is available at the end of the article
}

diagnosed with extensive-stage disease [3], which is defined as disease dissemination beyond the ipsilateral hemithorax including malignant pleural or pericardial effusion or hematogenous metastases [4]. Over the past 20 years, the standard therapy for most patients with extensive-staged small cell lung cancer (ES-SCLC) has been either carboplatin or cisplatin in combination with etoposide (EP) [5]. In 2002, the Japan Clinical Oncology Group (JCOG-9511) first acquired evidence for superior outcomes following therapy with irinotecan in combination with cisplatin (IP). Nevertheless, a subsequent

(c) The Author(s). 2018 Open Access This article is distributed under the terms of the Creative Commons Attribution 4.0 International License (http://creativecommons.org/licenses/by/4.0/), which permits unrestricted use, distribution, and reproduction in any medium, provided you give appropriate credit to the original author(s) and the source, provide a link to the Creative Commons license, and indicate if changes were made. The Creative Commons Public Domain Dedication waiver (http://creativecommons.org/publicdomain/zero/1.0/) applies to the data made available in this article, unless otherwise stated. 
and larger study failed to validate the observed difference survival benefit in JCOG-9511 between the IP and EP treatment arms. In 2010, in a meta-analysis, Jiang et al. [6] concluded that IP may have an advantage in overall response and OS as compared to EP in patients with ES-SCLC, but did not find superior results in progression-free survival (PFS); however, the authors did not include ethnicity in their analysis. Therefore, our meta-analysis was performed based on these prior studies to compare the efficacies and toxicities of IP and EP in patients with ES-SCLC, and these parameters were further analyzed in patient subpopulations.

\section{Methods}

\section{Search strategy and study selection}

The Cochrane Library, PubMed, and Embase electronic databases were used to perform an electronic search by combining following words: "small cell lung cancer" or "small cell lung carcinoma," "irinotecan" or "CPT-11," and "etoposide" or "VP-16". To limit publication bias, the search was limited to "randomized controlled trial" and no language, publishing time limitation, or other restrictions were imposed. We also searched the Physician Data Query registry of ClinicalTrials.gov (http://clinicaltrials.gov) to identify ongoing studies.

\section{Inclusion and exclusion criteria}

Two reviewers (Fei Xu and Xiaoli Ren) independently reviewed all studies that met the following selection criteria: (1) all patients recruited in the study who were diagnosed SCLC were previously untreated; (2) the study compared IP regimens with EP regimens; and (3) the study was a randomized controlled clinical trial. Trials were excluded if they did not meet the above inclusion criteria. Disagreements were resolved by discussion or by consulting with a third reviewer.

\section{Information extraction and assessment of methodological quality}

Two reviewers (Fei $\mathrm{Xu}$ and Xiaoli Ren) independently extracted the following information from the included studies: first author's name, year of publication, country, sex, average age, number of patients, chemotherapy regimens, stage of disease, primary endpoint, and second endpoint as well as hazard ratios (HRs) and respective confidence intervals for OS and PFS, complete response, partial response, overall response rate (ORR), disease control rate (DCR), 1-year survival rate, and 2-year survival rate. If HRs were not available, we extracted vital data through survival curves using Engauge Digitizer Version 4.1 software and then calculated HRs by the Tierney method [7]. Common adverse events of grade
3-4 toxicity such as anemia, leucopenia, neutropenia, thrombocytopenia, diarrhea, febrile neutropenia, infection, alopecia, fatigue and drug-related death were also extracted according to National Cancer Institute-Common Toxicity Criteria.

Methodological quality was assessed independently according to the following items: random sequence generation, allocation concealment, blinding of participants and personnel, blinding of outcome assessment, incomplete outcome data, selective reporting, and other bias. Each item was judged as "low," "high," or "unclear." Disagreements were resolved by discussion or consulting with a third reviewer.

\section{Statistical analysis}

Review manager 5.3 was used to analyze and generate data. Heterogeneity was identified using a chi-square test, and $\mathrm{I}^{2}\left(P<0.1\right.$ and $\left.\mathrm{I}^{2}>50 \%\right)$ indicated significant heterogeneity. In the event that obvious heterogeneity was deemed valid, the random-effects model was used. Otherwise, the fixed-effects model was employed. The HR was used for PFS and OS. For dichotomous data, relative risk (RR) was used for ORR, DCR, 1-year survival, 2-year survival, and adverse event data. A $P$ value $<0.05$ was considered statistically significant. $\mathrm{HR}>1$ reflects more deaths or progression in the EP arm. RR > 1 reflects more events in the IP arm.

\section{Results}

\section{Identification of studies and study quality}

We identified 1061 patient records, and seven clinical trials were identified on ClinicalTrials.gov according to the search strategy. After excluding duplicates, ongoing trials, trials of unknown status and results, and after screening titles and abstracts, 40 records were selected for full-text screening, of which nine publications [8-16] including 2451 patients that fulfilled all inclusion criteria were considered for analysis. A flow chart of our study is shown in Fig. 1. All identified studies were phase III randomized controlled trials. The included publications used cisplatin with two exceptions: Hermes et al. and Schmittel et al. used carboplatin. Detailed baseline characteristics of the included studies are presented in Table 1. According to the tool described in the Cochrane Handbook for Systematic Reviews of Interventions [17], we assessed the methodological quality of each included study (Figs. 2 and 3).

\section{Overall survival}

HRs for OS data were available for eight trials that altogether included 2390 patients (when data was acquired indirectly, HR was calculated by the Tierney method). The pooled $\mathrm{HR}$ was 0.85 , indicating that an 


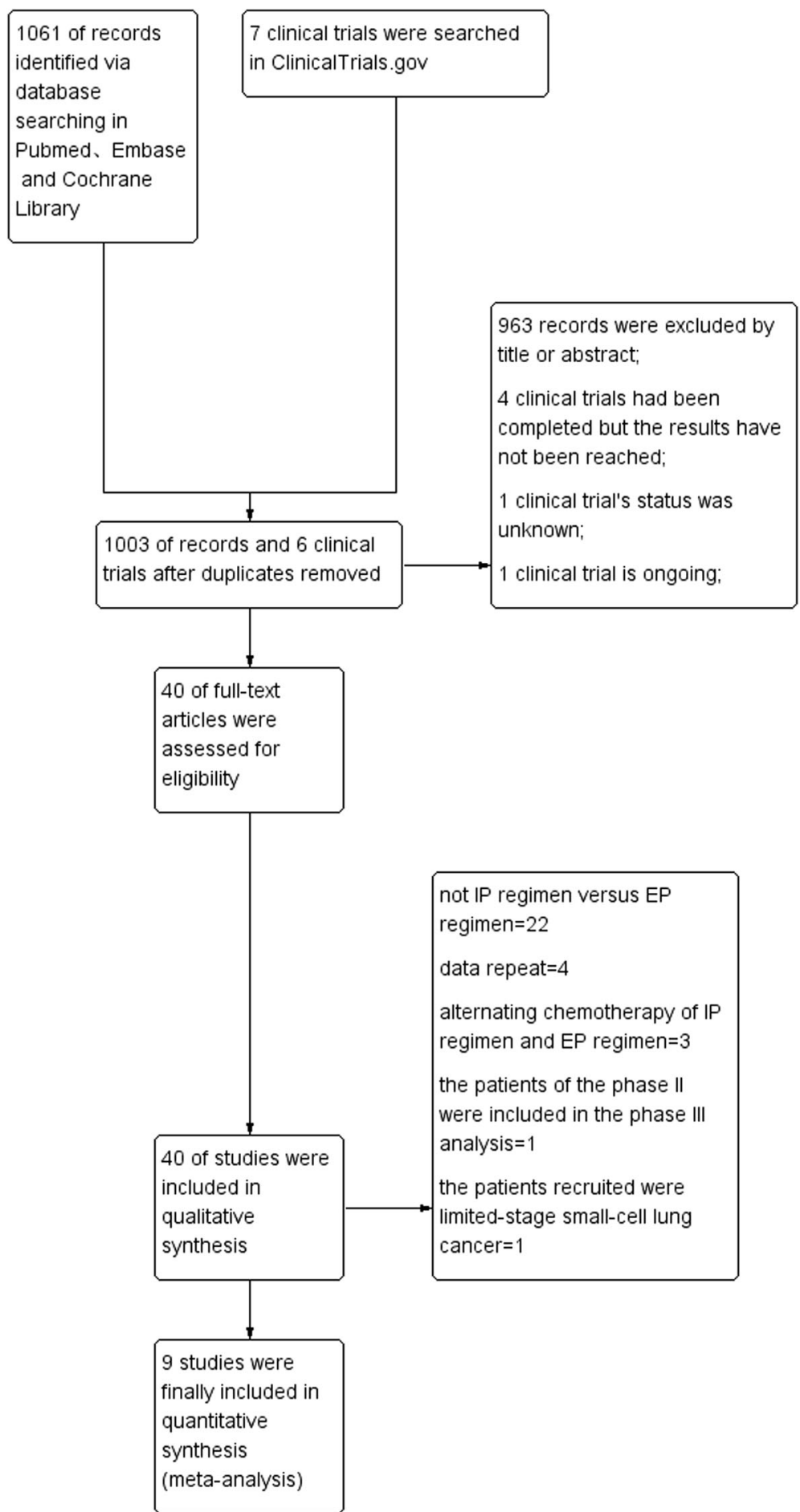

Fig. 1 Flow chart showing the progression of trials through the review

IP regimen likely prolongs OS in patients with SCLC $(\mathrm{HR}=0.85 ; 95 \% \mathrm{CI}, 0.78-0.92 ; \mathrm{P}<0.0001 ;$ Fig. 4$)$. The heterogeneity test $\left(\mathrm{Chi}^{2}=9.65 ; \quad P=0.21 ; \quad \mathrm{I}^{2}=27 \%\right)$ indicated that mild heterogeneity was present among the included studies; thus, the fixed-effects model was used. Although no significant heterogeneity was 


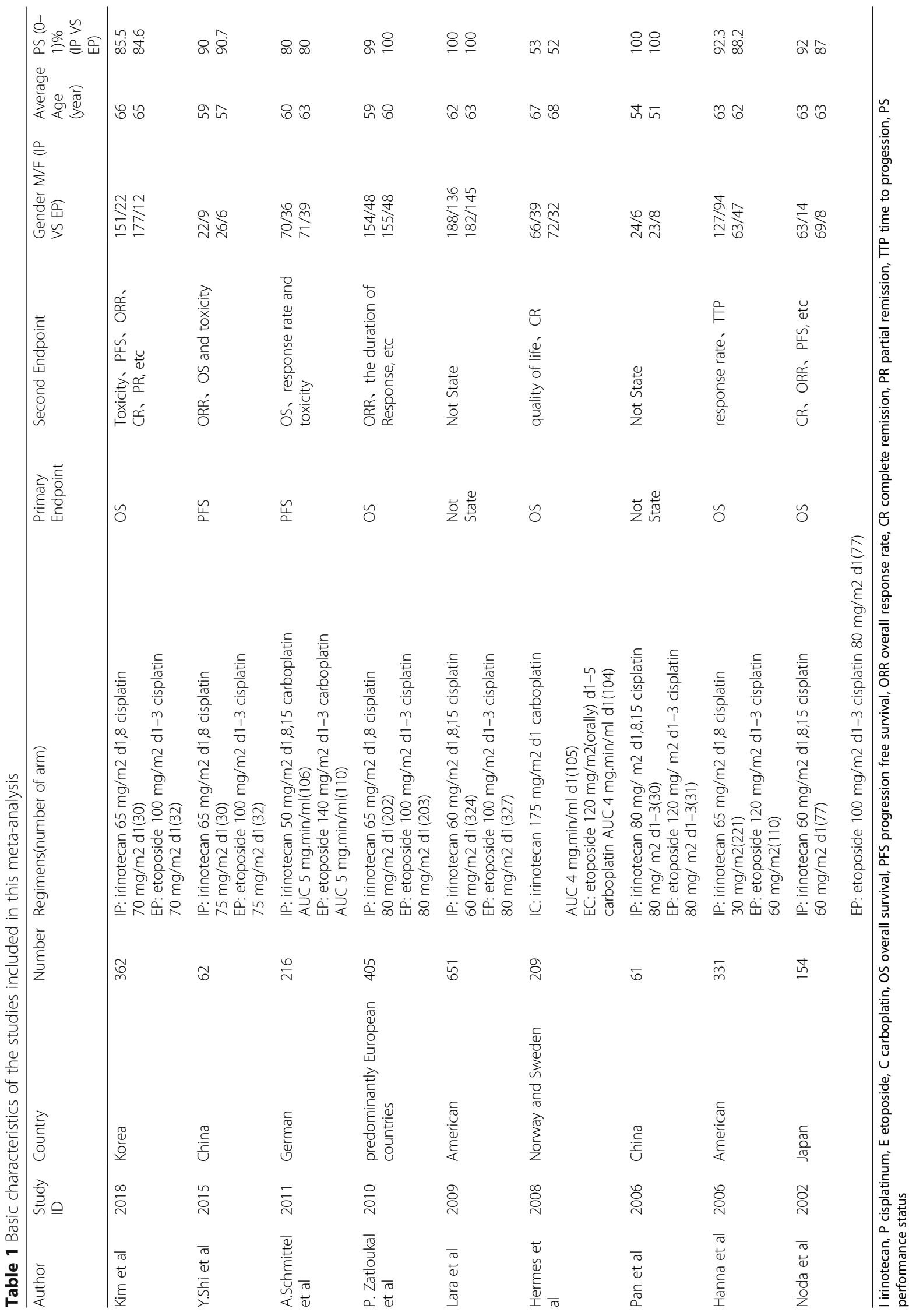




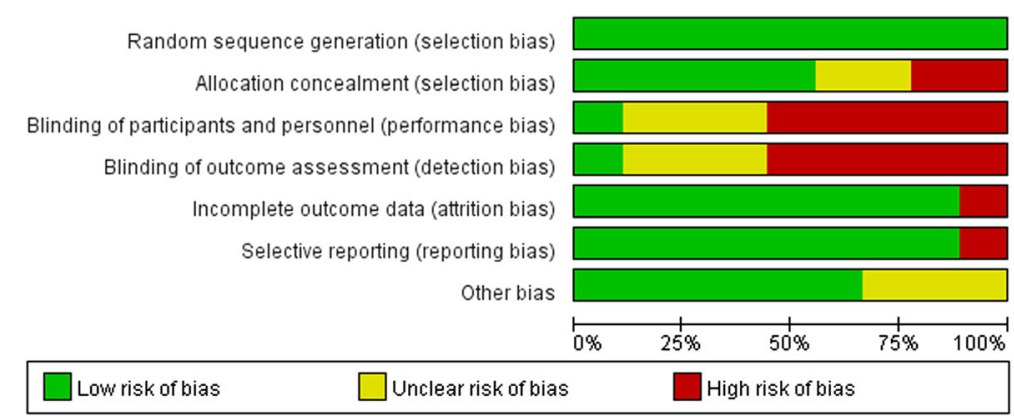

Fig. 2 Risk of bias graph

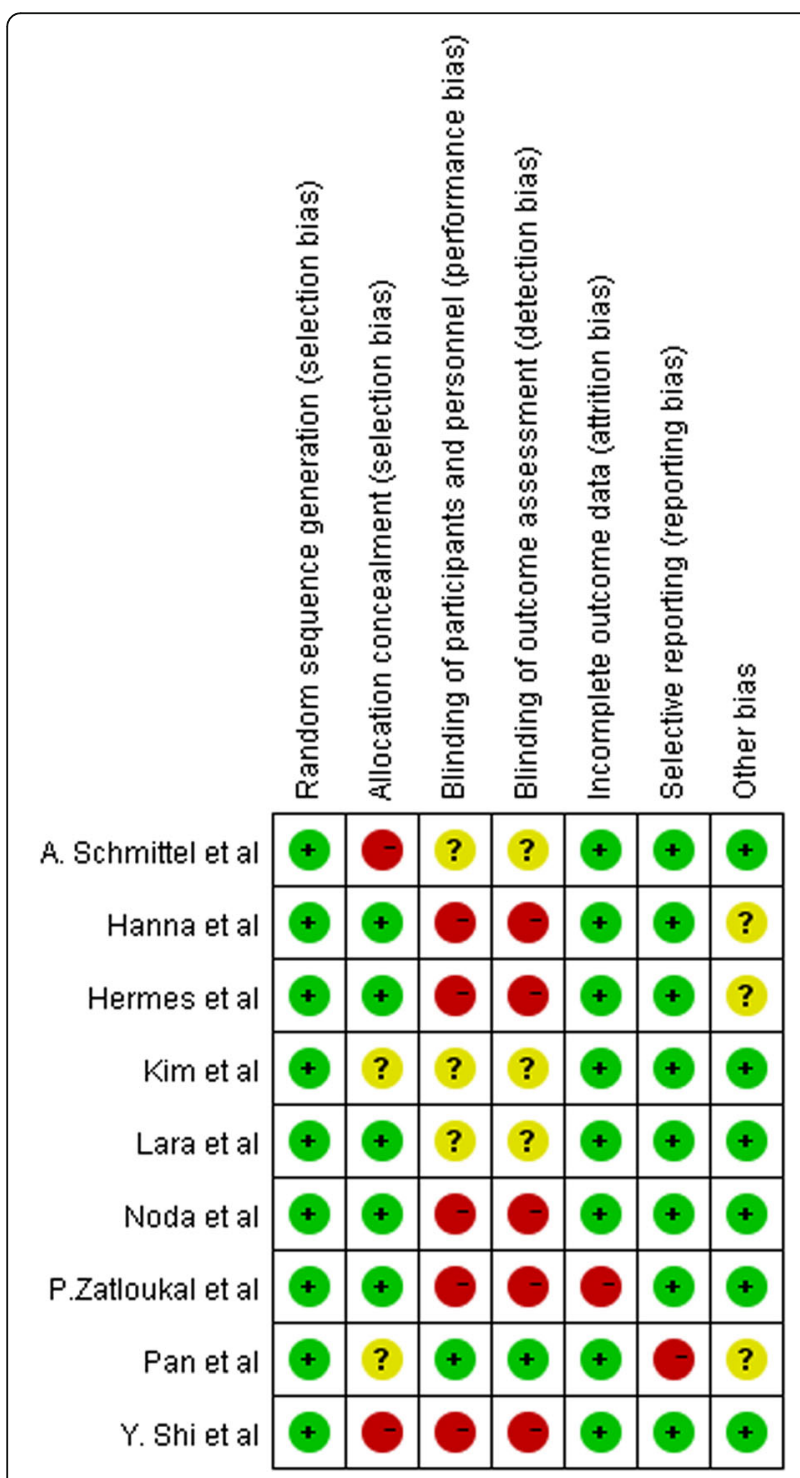

Fig. 3 Risk of bias summary observed in this comparison, we performed subgroup analyses stratified by the use of platinum and patient ethnicity (Asian or non-Asian), and sensitivity analysis was employed to explore sources of heterogeneity. The details of subgroup analysis are listed in Table 2 . We did not find obvious differences in heterogeneity, with one following exception: when the study performed by Noda et al. was excluded, heterogeneity declined from $\mathrm{I}^{2}=27 \%$ to $\mathrm{I}^{2}=0 \%$ (Fig. 5).

\section{Progression-free survival}

HR for PFS was available for seven trials that included 2181 patients. The pooled HR for PFS was 0.88 (95\% CI, 0.82-0.96; $P=0.002$ ), and which was statistically significant. The fixed-effects model was adopted due to the mild heterogeneity $\left(\mathrm{Chi}^{2}=10.77\right.$; $\left.P=0.10 ; I^{2}=44 \%\right)$. The results of subgroup analysis stratified by ethnicity (non-Asian or Asian patients) are shown in Fig. 6. We also performed sensitivity analysis and found that heterogeneity declined from $\mathrm{I}^{2}=44 \%$ to $\mathrm{I}^{2}=9 \%$ when the study performed by Noda et al. was excluded, however, the outcome was nearly unchanged (Fig. 7).

\section{Overall response, disease control, 1-year survival and 2-} year survival rates

Data concerning overall response rate (ORR), disease control rate (DCR), 1-year survival rate, and 2-year survival rate were separately available for eight, seven, four, and three studies, respectively. The pooled RR of ORR was 1.08 (95\% CI, 1.001.16; $P=0.05)$, which was statistically significant (Fig. 8). Heterogeneity was mild $\left(\mathrm{Chi}^{2}=10.92 ; P=\right.$ $\left.0.14 ; I^{2}=36 \%\right)$. The RR of the subgroup analysis with Asian patients was 1.23 (95\% CI, 1.10-1.39) and was 1.01 with non-Asian patients $(95 \% \mathrm{CI}$, 0.92-1.11). Significant discrepancies in RR of DCR and 1-year survival rate were not detected (Table 3 ). 


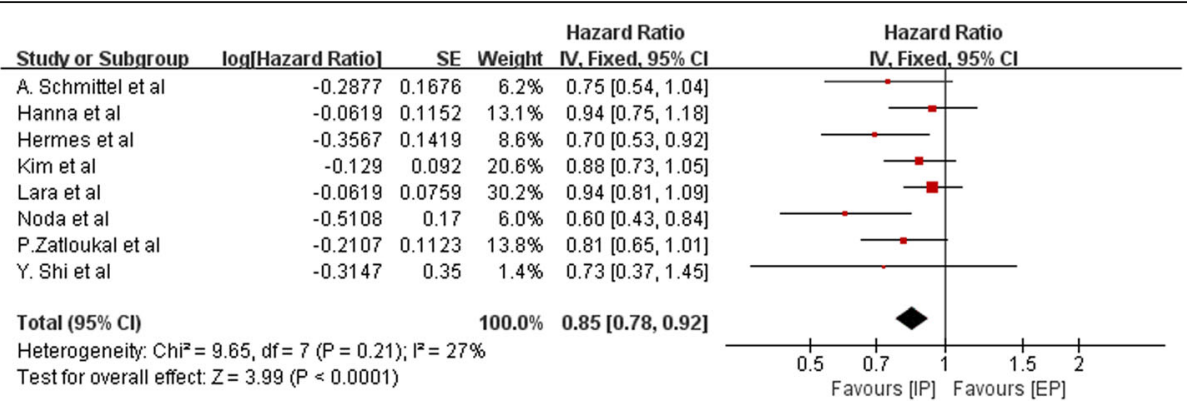

Fig. 4 Forest plots estimating OS in IP vs EP

It is notable that the $\mathrm{RR}$ of the 2-year survival rate was 1.77 (95\% CI $1.19-2.63 ; P=0.01$ ).

\section{Adverse effects \\ Hematological toxic effects}

Data on the frequency of National Cancer Institute Common Toxicity Criteria (NCI-CTC) grade 3-4 hematologic toxic effects, such as anemia, leucopenia, neutropenia, thrombocytopenia, and febrile neutropenia, were available from three to nine studies. Figures 9, 10, 11, 12 and 13 summarize the toxicity results. Patients treated with EP regimens were at a higher risk of grade 3-4 anemia (pooled $\mathrm{RR}=0.76$; 95\% CI, 0.54-1.09; $P=0.13)$, grade $3-4$ leucopenia (pooled $\mathrm{RR}=0.58 ; 95 \% \mathrm{CI}, \quad 0.44-0.77 ; \quad P=0.0002$ ), grade 3-4 neutropenia (pooled $\mathrm{RR}=0.60 ; 95 \% \mathrm{CI}$, $0.46-0.77 ; \quad P<0.0001)$, grade $3-4$ thrombocytopenia (pooled RR $=0.46 ; 95 \% \mathrm{CI}, 0.31-0.70, P=0.0003$ ), and grade $3-4$ febrile neutropenia (pooled $R R=0.64 ; 95 \%$ CI, 0.42-0.97; $P=0.03)$. Due to the heterogeneity regarding grade $3-4$ anemia $\left(\mathrm{Tau}^{2}=0.16 ; \mathrm{Chi}^{2}=19.98\right.$; $\left.P=0.01 ; \mathrm{I}^{2}=60 \%\right)$, grade $3-4$ leucopenia $\left(\mathrm{Tau}^{2}=0.08\right.$; $\left.\mathrm{Chi}^{2}=15.44 ; \quad P=0.02 ; \mathrm{I}^{2}=61 \%\right)$, grade $3-4$ neutropenia $\left(\mathrm{Tau}^{2}=0.09 ; \mathrm{Chi}^{2}=53.49 ; \mathrm{P}<0.00001 ; \mathrm{I}^{2}=89 \%\right)$, grade 3-4 thrombocytopenia $\left(\mathrm{Tau}^{2}=0.22 ; \mathrm{Chi}^{2}=\right.$ 21.69; $P=0.006 ; \mathrm{I}^{2}=63 \%$ ), and grade $3-4$ febrile neutropenia $\left(\mathrm{Tau}^{2}=0.14 ; \mathrm{Chi}^{2}=11.13 ; P=0.05 ; \mathrm{I}^{2}=55 \%\right)$ were obvious, the random-effects models were used.

\section{Non-hematological toxic effects}

All trials reported grade 3-4 diarrhea, seven reported infection, four reported fatigue, and three reported alopecia and drug-related deaths. Figures 14 and 15 presented the results of grade 3-4 diarrhea and infection. An IP chemotherapy regimen led to more grade 3-4 diarrhea (pooled $\mathrm{RR}=7.96$ 95\% CI, 5.21-12.17; $\mathrm{P}<0.00001$ ) and less infection (pooled $\mathrm{RR}=0.80 ; 95 \% \mathrm{CI}, 0.67-0.95 ; \mathrm{P}=0.01)$. On the other hand, differences in the incidence of alopecia (pooled $\mathrm{RR}=0.48 ; \quad 95 \% \quad \mathrm{CI}, \quad 0.18-1.29 ; \quad P=0.15), \quad$ fatigue (pooled $\mathrm{RR}=1.18 ; 95 \% \mathrm{CI}, 0.98-1.42 ; P=0.07$ ), and drug-related death (pooled $\mathrm{RR}=1.53 ; 95 \% \mathrm{CI}, 0.79$ 2.99; $P=0.21$ ) were not statistically significant between patients treated with an IP regimen as compared to those who were treated with an EP regimen. The details of all the toxic effects were illustrated in Table 4.

\section{Discussion}

Chemotherapy is an essential component of appropriate treatment for patients with SCLC [18]. The current standard treatment is chemotherapy with or without local radiotherapy for patients with SCLC who have a good performance status (0-2), as recommended by the National comprehensive cancer network guidelines as category 1 evidence. EP is the most commonly used chemotherapy regimen. This

Table $\mathbf{2}$ The outcome of subgroup analysis stratified by platinum regimen and ethnicity

\begin{tabular}{llllll}
\hline Subgroups & Pooled HR & $95 \% \mathrm{Cl}$ & $P$-Value & $\mathrm{I}^{2}$ For Homogeneity & Total \\
\hline Cisplatin & 0.87 & $0.80-0.95$ & 0.002 & $28 \%$ & $\mathrm{HR}=0.85,95 \% \mathrm{Cl} 0.78-0.92, \mathrm{P}<0.0001, \mathrm{I}^{2}=27 \%$ \\
carboplatin & 0.72 & $0.58-0.89$ & 0.002 & $0 \%$ & \\
Asian people & 0.80 & $0.69-0.94$ & 0.005 & $50 \%$ & \\
non-Asian people & 0.86 & $0.79-0.95$ & 0.003 & $20 \%$ & \\
\hline
\end{tabular}




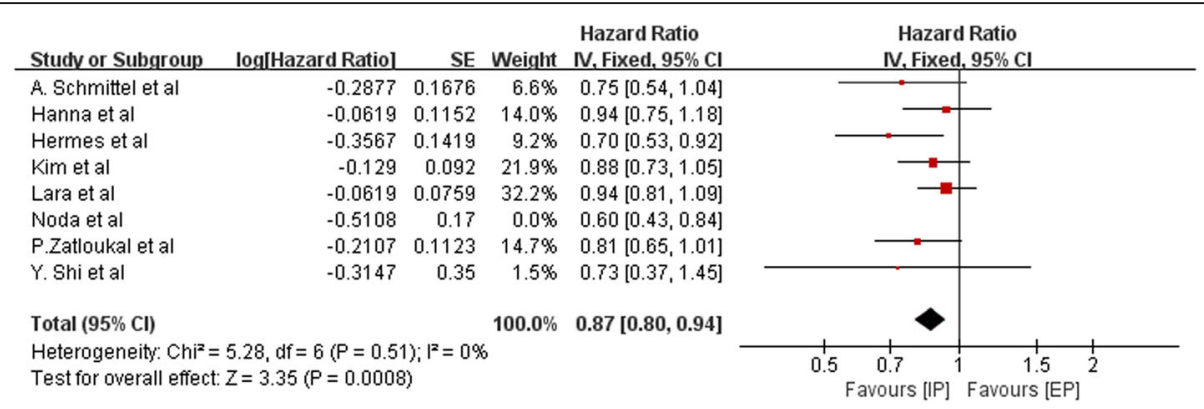

Fig. 5 Sensitivity analysis of OS was employed to explore sources of heterogeneity. Heterogeneity declined from $I^{2}=27 \%$ to $\left.\right|^{2}=0 \%$ when the study performed by Noda et al. was excluded

regimen provides response rates of $60 \%$ to $80 \%$, with a median survival time of 8 to 10 months. Thus, chemotherapeutic agents with greater activity are urgently needed.

JCOG previously reported the results of a randomized phase III trial (JCOG9511). They found that irinotecan, an inhibitor of the nuclear enzyme topoisomerase I, could improve OS and PFS when combined with platinum. Nevertheless, a series of studies conducted in America and Europe failed to confirm these positive results [9-12]. More rigorous studies were included in this meta-analysis to further compare efficacy and toxicity between IP and EP regimens; we subsequently analyzed the combined results thereof within the various subgroups.

In this meta-analysis, IP and EP regimens were compared in terms of OS, PFS, ORR, DCR, 1-year survival rate, 2-year survival rate, and common toxic adverse events. We found that an IP regimen significantly improves OS as compared to an EP regimen in ED-SCLC patients. When stratifying subgroup analysis by platinum type and ethnicity, OS results were consistent with the overall results. However, we found that the HRs were lower in patients treated with carboplatin and in Asian patients. These data indicate that irinotecan is superior to etoposide in combination with carboplatin-based chemotherapy, and that Asian patients receive a greater benefit from an IP regimen.

The OS of the patients who received follow-up treatment could be influenced and this may explain the inconspicuous superior result. PFS as a more meaningful measure of treatment effects, a superior outcome of IP treatment was found. That is to say, the IP regimen showed a increase in PFS, and the difference was statistically significant. When we performed subgroup analysis stratified by ethnicity, we found that the HR for Asian patients was 0.79 , which was statistically significant $(P=$ 0.002, 95\% CI, 0.68-0.92). The HR for non-Asian patients was $0.92(95 \% \mathrm{CI}, 0.84-1.01)$, indicating that the IP and EP regimens led to comparable PFS in this subgroup. This is probably because a reduction of irinotecan often occurs in non-Asian patients who more frequently

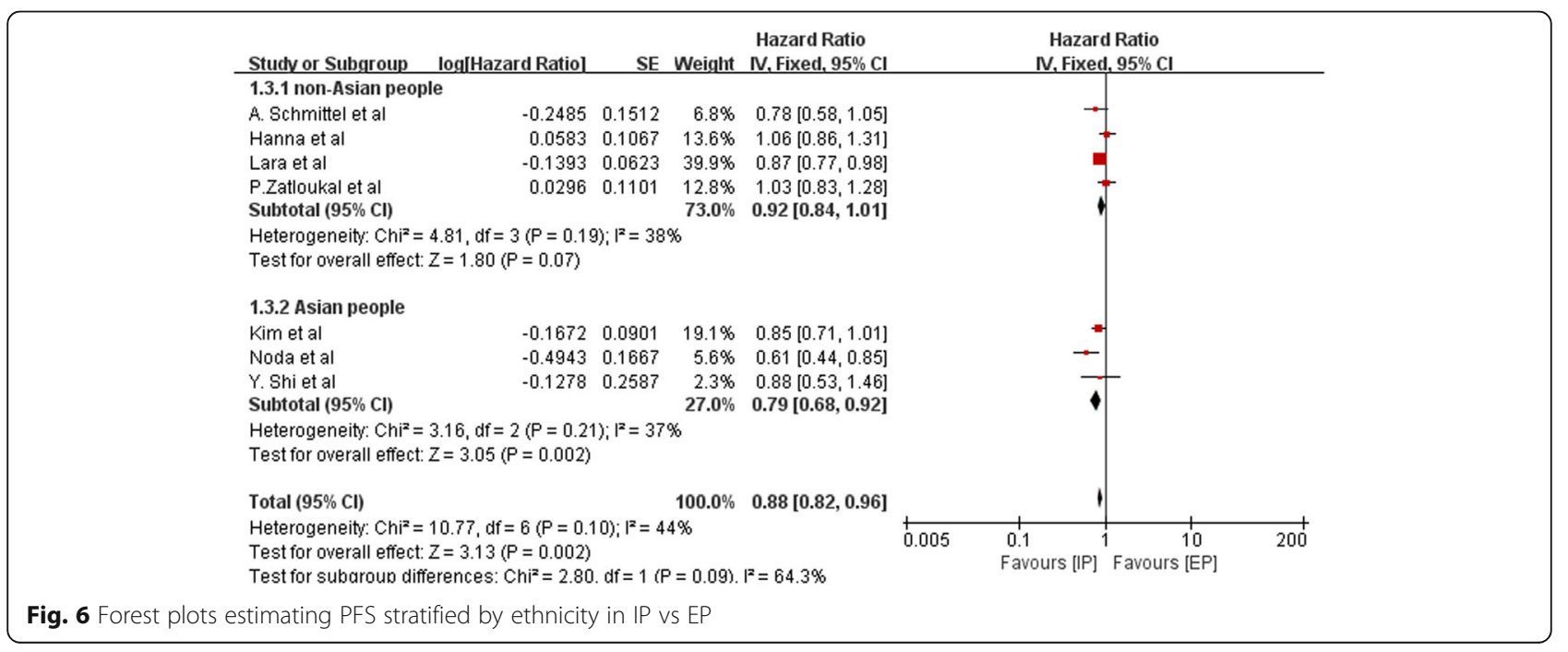




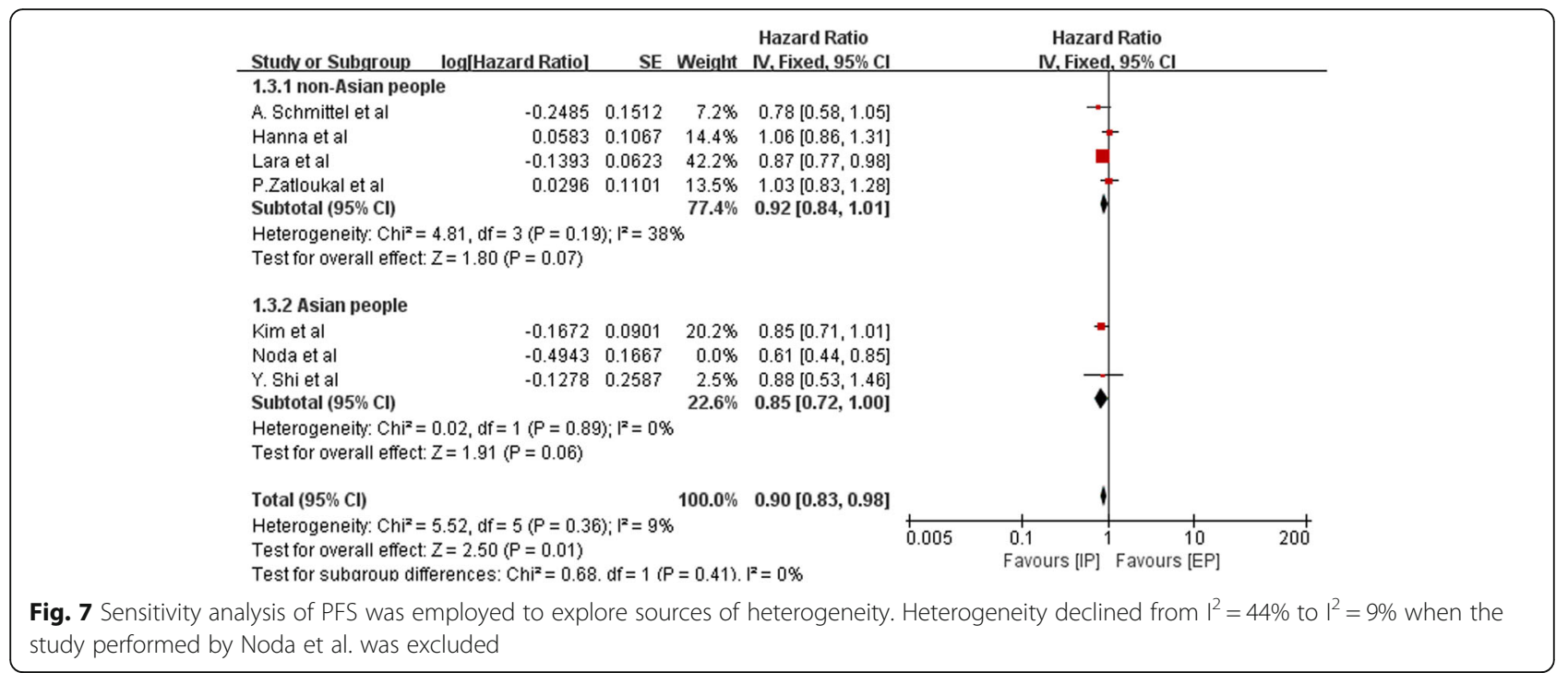

carry the UGT1A1*28 allele and are thus at an increased risk for severe diarrhea $[19,20]$. Thus, the efficacy of irinotecan might be influenced by dose reduction in non-Asian patients.

Sensitivity analysis was performed excluding the Noda trial (JCOG9511), which prematurely concluded after interim analysis because they found significant differences in OS, and reduced heterogeneity (in OS: $P=0.51, \mathrm{I}^{2}=0 \%$; in PFS: $P=0.36, \mathrm{I}^{2}=9 \%$ ). The HRs, which were 0.87 for OS $(95 \% \mathrm{CI}, 0.80-0.94 ; P=$ 0.0008 ) and 0.90 for PFS (95\% CI, 0.83-0.98; $P=$ 0.01 ), were almost in line with the overall results. In addition, a different extent of dose reduction was present in each study. Therefore, we conclude that the trial conducted by Noda et al. (JCOG9511) and the various doses of chemotherapy regimens used in various countries might account for some of the observed heterogeneity in our meta-analysis.

That the pooled RR showed superior ORR of IP regimen implies that more patients will respond to chemotherapy when treated with an IP regimen, especially for Asian patients. Differences in DCR and 1-year survival rate were not statistically significant. Moreover, we found that irinotecan was superior to etoposide in 2-year survival rate. However, the outcome of relatively higher RR for 2-year survival rate warrants further discussion due to the low number of studies and recruited patients.

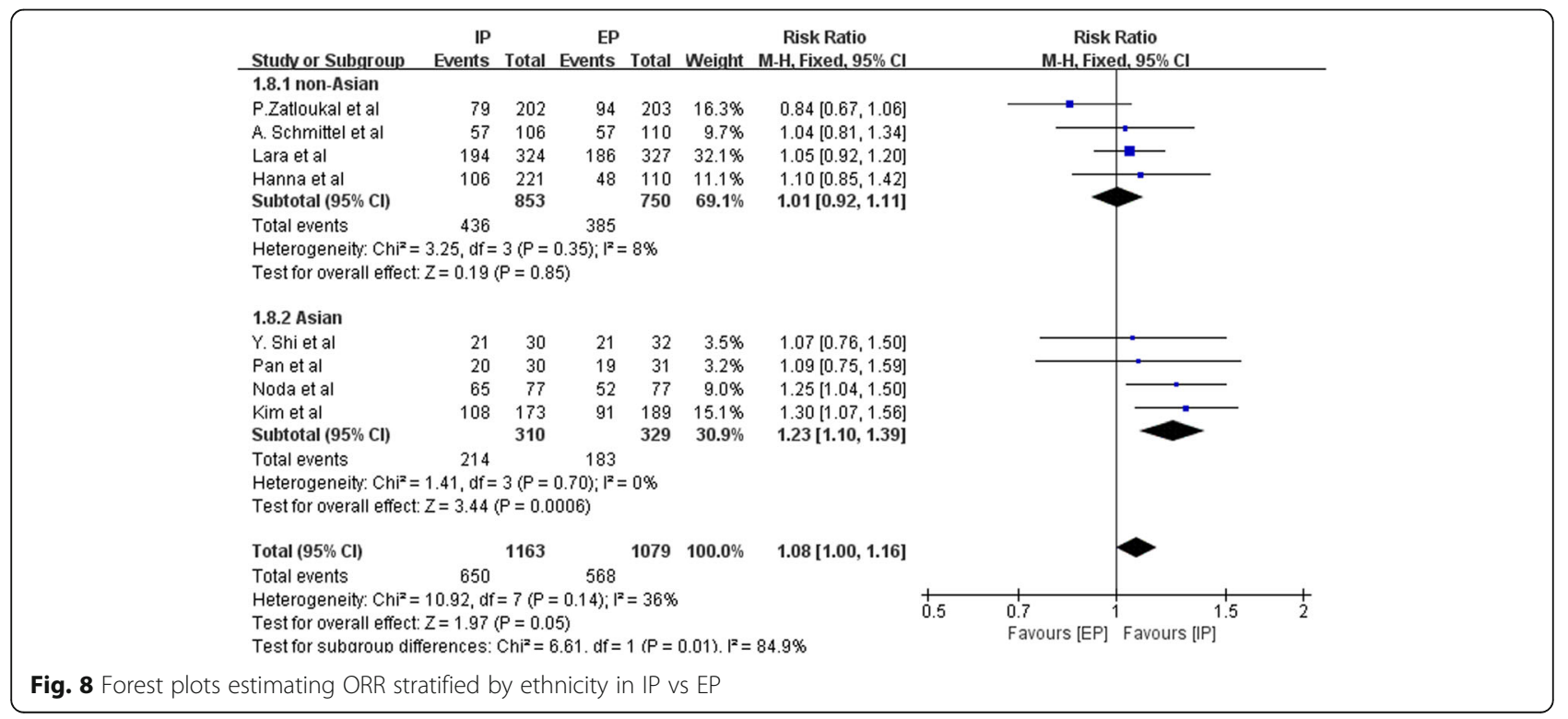


Table 3 The outcomes of RR, 95\% Cl, and $\mathrm{I}^{2}$ in CR, PR, ORR, DCR, 1-year survival rate, and 2-year survival rate

\begin{tabular}{llllll}
\hline Analysis & Number Of Concerning Trials & Pooled RR & $95 \% \mathrm{Cl}$ & P-Value & I $^{2}$ For Homogeneity \\
\hline CR & 7 & 1.49 & $0.95-2.33$ & 0.08 & $27 \%$ \\
PR & 6 & 1.08 & $0.88-1.32$ & 0.47 & $68 \%$ \\
ORR & 8 & 1.08 & $1.00-1.16$ & 0.05 & $36 \%$ \\
DCR & 7 & 1.02 & $0.96-1.08$ & 0.49 & $0 \%$ \\
1-Year Survival Rate & 4 & 1.11 & $0.96-1.28$ & 0.18 & $0 \%$ \\
2-Year Survival Rate & 3 & 1.77 & $1.19-2.63$ & 0.005 & $42 \%$ \\
\hline
\end{tabular}

DCR disease control rate

Toxicity analyses indicated that more patients treated with an IP regimen were likely to experience grade 3-4 diarrhea, and fewer experienced grade 3-4 hematologic toxic effects than those treated with an EP regimen. These results are in agreement with those of previous studies and the meta-analysis of safety of IP and EP [21]. We also performed subgroup analysis to explore diarrhea as an adverse event. The pooled RR in Asian patients was 5.93 (95\% CI, 2.67-13.16; $\mathrm{P}<0.0001)$ and 8.74 in non-Asian patients (95\% CI, 5.30-14.41; $P<$ $0.00001)$. This indicates that non-Asian patients are more likely to experience grade 3-4 diarrhea. However, the difference was not statistically significant $\left(\mathrm{Chi}^{2}=0.65 ; \mathrm{df}=1 ; \quad P=0.42 ; \mathrm{I}^{2}=0 \%\right)$. This difference occurred might because the aforementioned UGT1A1*28 genotype, which bears a lower allele frequency in Asians than in Caucasians [19], confers a marked increase in irinotecan-induced grade 3-4 diarrhea [20]. Thus, a dose reduction of irinotecan is more likely to occur in Caucasians. Meanwhile some in vitro studies indicated that gene polymorphisms in the UGT1A1*6 gene were also associated with irinotecan metabolism [22, 23]. The frequency of the UGT1A1*6 mutant genotype was higher in Asian patients than in Caucasians [22]. A meta-analysis by Cheng et al. demonstrated that the heterozygous variant of UGT1A1*6 showed no significant risk for severe diarrhea, while there was a significant risk associated with the homozygous variant [24].
Therefore, we speculate that the UGT1A1*6 gene polymorphism may have an impact on the development of irinotecan-induced diarrhea in the Asian population. Confounding factors, such as differing doses of irinotecan, and the UGT1A1 gene polymorphism may be the reasons why there was no significant association between ethnicities and development of grade 3-4 diarrhea in populations.

We believe that the strength of this study lies in the fact that we conducted a quality assessment to guarantee that studies of a higher quality were included in the meta-analysis. Furthermore, we performed subgroup analyses of both ethnicity and platinum. Finally, the results were therefore more robust and reliable due to the consequence of sensitivity analysis.

A potential limitation of this meta-analysis is related to the different doses of chemotherapy regimens, and the performance status thereof in the included trials. A lack of information regarding the detailed dosage and performance status information for each of the groups meant that we could not perform the respective subgroup analyses. Another possible bias may have been introduced by the study conducted by Noda et al., which might lead to an overly optimistic result due to its premature conclusion. Additionally, more individual patient data were needed to conduct our meta-analysis, as extracting data from a survival curve inevitably introduced bias.

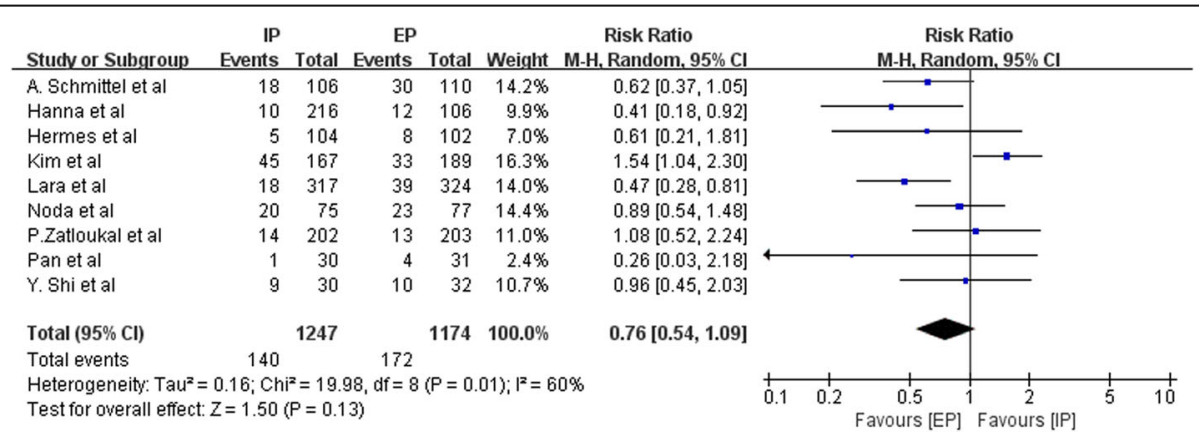

Fig. 9 Forest plots estimating grade $3-4$ anemia in IP vS EP 


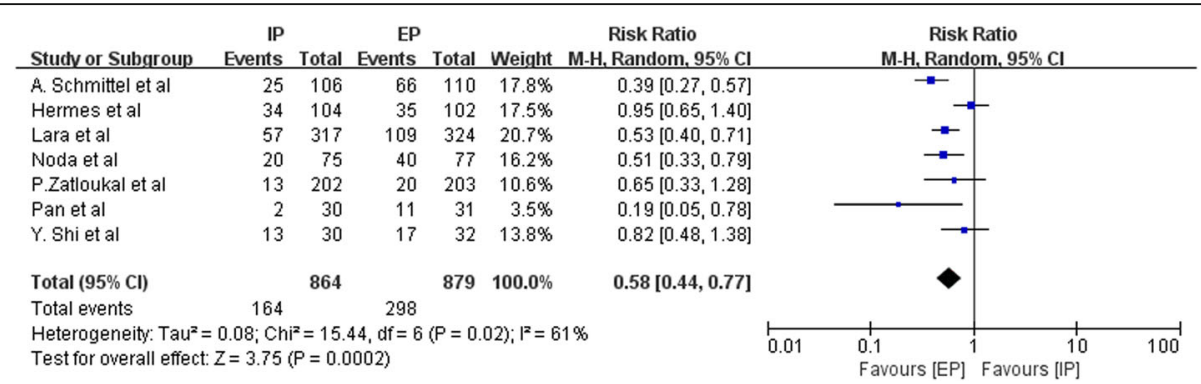

Fig. 10 Forest plots estimating grade 3-4 leucopenia in IP vS EP

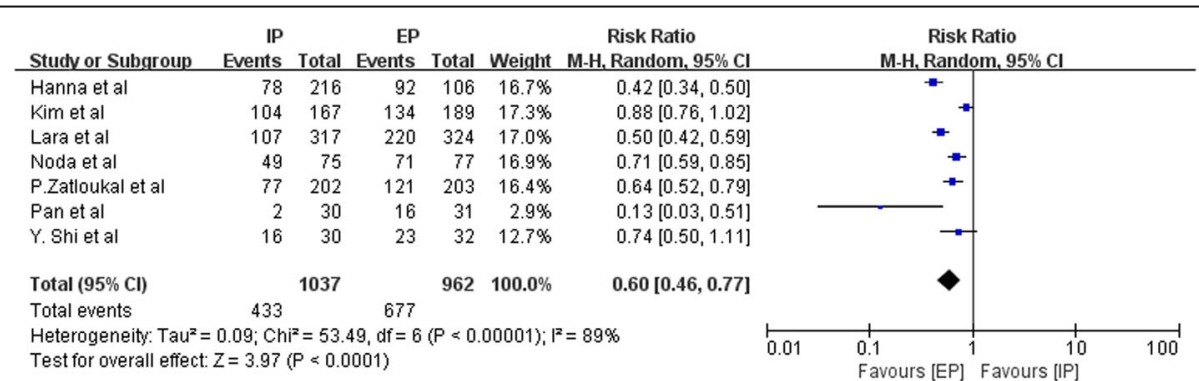

Fig. 11 Forest plots estimating grade 3-4 neutropenia in IP vs EP

\begin{tabular}{|c|c|c|c|c|c|c|c|c|c|c|}
\hline Studv or Subgroup & $\begin{array}{r}\text { IP } \\
\text { Events }\end{array}$ & Total & $\begin{array}{r}\text { EP } \\
\text { Events }\end{array}$ & Total & Weight & $\begin{array}{c}\text { Risk Ratio } \\
\text { M-H, Random, } 95 \% \mathrm{Cl}\end{array}$ & & $\begin{array}{r}\text { Risk F } \\
\text { M-H, Rando }\end{array}$ & $\begin{array}{l}\text { Ratio } \\
\text { om, } 95 \% \mathrm{Cl}\end{array}$ & \\
\hline A. Schmittel et al & 24 & 106 & 50 & 110 & $17.0 \%$ & $0.50[0.33,0.75]$ & & -- & & \\
\hline Hanna et al & 9 & 216 & 20 & 106 & $12.2 \%$ & $0.22[0.10,0.47]$ & & & & \\
\hline Hermes et al & 16 & 104 & 27 & 102 & $14.9 \%$ & $0.58[0.33,1.01]$ & & & & \\
\hline Kim et al & 21 & $16 ?$ & 25 & 189 & $15.1 \%$ & $0.95[0.55,1.63]$ & & & & \\
\hline Lara et al & 12 & 317 & 48 & 324 & $14.1 \%$ & $0.26[0.14,0.47]$ & & & & \\
\hline Noda et al & 4 & 75 & 14 & 77 & $8.7 \%$ & $0.29[0.10,0.85]$ & & & & \\
\hline P.Zatloukal et al & 11 & 202 & 9 & 203 & $10.8 \%$ & $1.23[0.52,2.90]$ & & & & \\
\hline Pan et al & 0 & 30 & 5 & 31 & $1.9 \%$ & $0.09[0.01,1.63]$ & & & & \\
\hline Y. Shi et al & 2 & 30 & 6 & 32 & $5.4 \%$ & $0.36[0.08,1.63]$ & & & & \\
\hline Total $(95 \% \mathrm{Cl})$ & & 1247 & & 1174 & $100.0 \%$ & $0.46[0.31,0.70]$ & & & & \\
\hline Total events & 99 & & 204 & & & & & & & \\
\hline \multicolumn{7}{|c|}{$\begin{array}{l}\text { Heterogeneity: } \text { Tau }^{2}=0.22 ; \mathrm{Ch}^{2}=21.69, \mathrm{df}=8(\mathrm{P}=0.006) ;\left.\right|^{2}=63 \% \\
\text { Test for overall effect: } Z=3.64(P=0.0003)\end{array}$} & 0.01 & $\begin{array}{l}0.1 \\
\text { Favours [EP] }\end{array}$ & $\begin{array}{r}10 \\
\text { Favours [IP] }\end{array}$ & 100 \\
\hline
\end{tabular}

Fig. 12 Forest plots estimating grade $3-4$ thrombocytopenia in IP vS EP

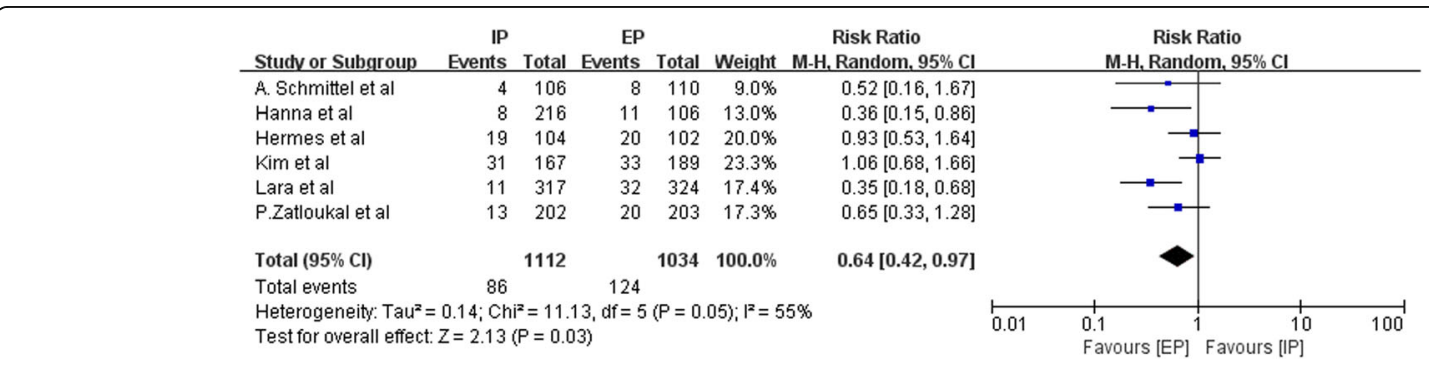

Fig. 13 Forest plots estimating grade 3-4 febrile neutropenia in IP VS EP 


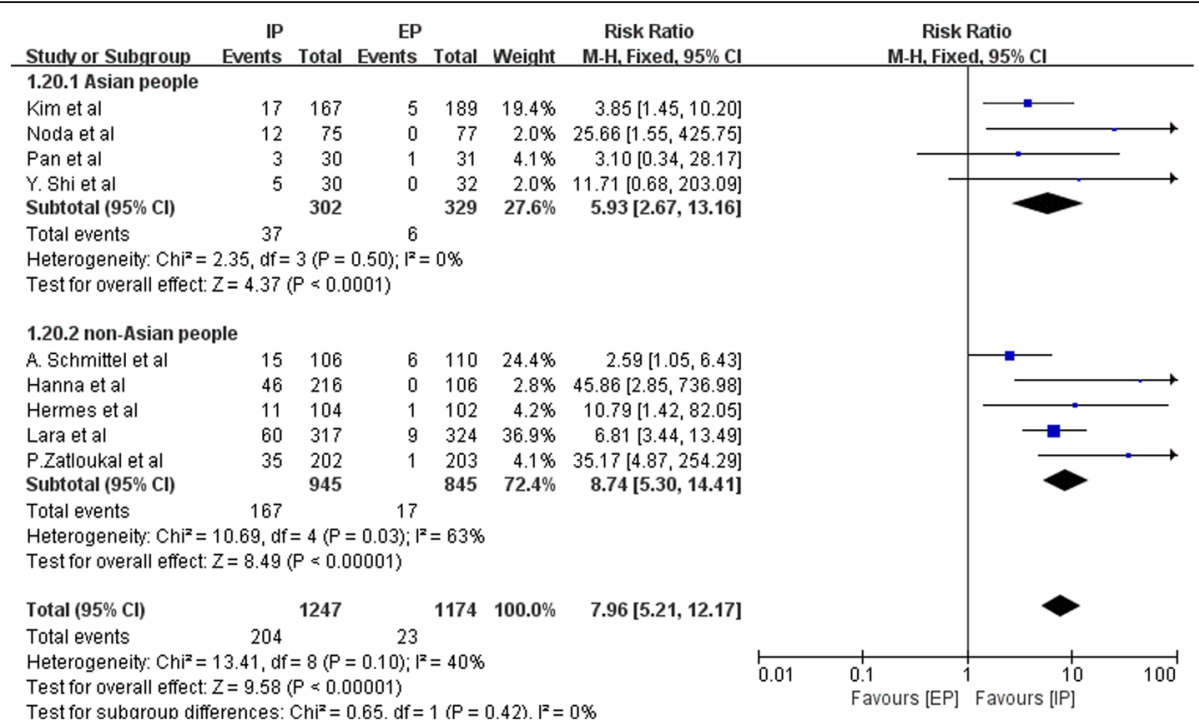

Fig. 14 Forest plots estimating grade 3-4 diarrhea stratified by ethnicity in IP VS EP

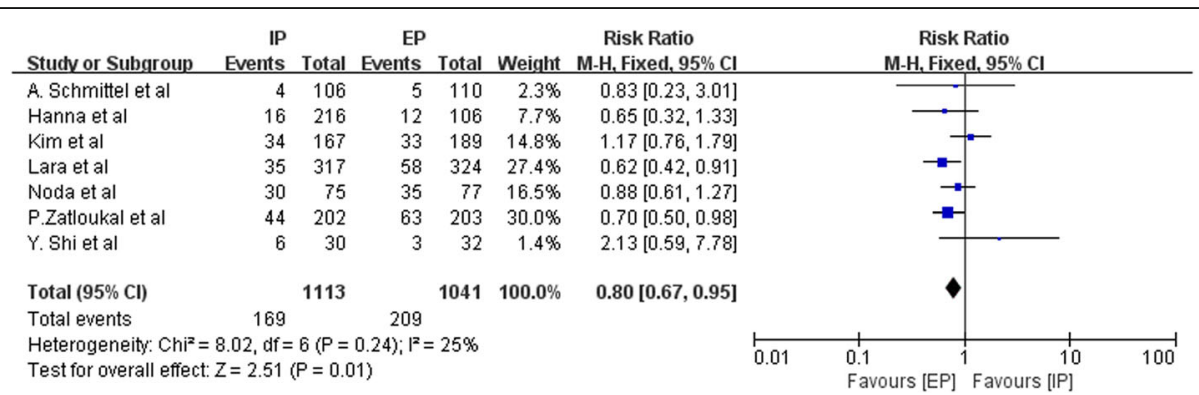

Fig. 15 Forest plots estimating infection in IP vs EP

Table 4 Toxicity outcomes in this meta-analysis

\begin{tabular}{|c|c|c|c|c|c|c|}
\hline Adverse Effects & & $\begin{array}{l}\text { Number Of } \\
\text { Concerning Trials }\end{array}$ & Pooled RR & $95 \% \mathrm{Cl}$ & P-Value & $1^{2}$ For Homogeneity \\
\hline \multirow[t]{5}{*}{ Hematological Toxic Effects } & Grade 3-4 Anemia & 9 & 0.76 & $0.54-1.09$ & 0.13 & $60 \%$ \\
\hline & Grade 3-4 Leucopenia & 7 & 0.58 & $0.44-0.77$ & 0.0002 & $61 \%$ \\
\hline & Grade 3-4 Neutropenia & 7 & 0.60 & $0.46-0.77$ & $<0.0001$ & $89 \%$ \\
\hline & Grade 3-4 Thrombocytopenia & 9 & 0.46 & $0.31-0.70$ & 0.0003 & $63 \%$ \\
\hline & Grade 3-4 Febrile Neutropenia & 6 & 0.64 & $0.42-0.97$ & 0.03 & $55 \%$ \\
\hline \multirow[t]{5}{*}{ Non-hematological Toxic Effects } & Grade 3-4 Diarrhea & 9 & 7.96 & $5.21-12.17$ & $<0.00001$ & $40 \%$ \\
\hline & Infection & 7 & 0.80 & $0.67-0.95$ & 0.01 & $25 \%$ \\
\hline & Alopecia & 3 & 0.48 & $0.18-1.29$ & 0.15 & $88 \%$ \\
\hline & Fatigue & 4 & 1.18 & $0.98-1.42$ & 0.07 & $0 \%$ \\
\hline & Drug-related Death & 3 & 1.53 & $0.79-2.99$ & 0.21 & $0 \%$ \\
\hline
\end{tabular}




\section{Conclusions}

In summary, for patients with ED-SCLC who have a poor prognosis, the question of which regimen to use is a relevant clinical issue requiring consideration of several factors. Given that IP regimens improved OS, PFS, and ORR as compared to EP regimens, particularly for Asian patients, we conclude that IP regimens can confer a survival benefit. Patients who were treated with an IP regimen experienced grade 3-4 diarrhea more frequently, including fatal diarrhea, and experienced fewer hematologic toxic events that were generally manageable and reversible with the application of corresponding symptomatic treatment drugs. For this reason, toxic events might be a vital factor in regimen selection. We conclude that IP regimens may substitute for EP regimens, particularly for ED-SCLC patients who have a good performance status.

\section{Abbreviations}

Cl: confidence interval; DCR: disease control rate; EP: Etoposide in combination with cisplatin; ES-SCLC: extensive-staged small cell lung cancer; HR: hazard ratio; IP: Irinotecan in combination with cisplastin; ORR: overall response rate; OS: overall survival; PFS: progression-free survival; RR: relative risk; SCLC: small cell lung cancer

\section{Acknowledgements}

I gratefully acknowledge my supervisor Professor Shu Xia for his help. I appreciate his patience, encouragement, and professional instructions during my thesis writing process.

\section{Availability of data and materials}

All data analysed during this study are included in this published article.

\begin{abstract}
Authors' contributions
FX and SX conceived and designed the study. FX and XLR performed the statistical analysis. QXL and YC (Yu Chen) carried out the literature search and data collection. YC (Yuan Chen) and RCL reviewed and edited the manuscript. All authors read and approved the manuscript.
\end{abstract}

\section{Ethics approval and consent to participate}

Not applicable.

\section{Consent for publication}

Not applicable.

\section{Competing interests}

The authors declare that they have no competing interests.

\section{Publisher's Note}

Springer Nature remains neutral with regard to jurisdictional claims in published maps and institutional affiliations.

\section{Author details}

${ }^{1}$ Department of Oncology, Tongji Hospital, Tongji Medical College of Huazhong University of Science and Technology, Wuhan, People's Republic of China. ${ }^{2}$ Department of Oncology, Affiliated Hospital of Hebei University of Engineering, Handan, People's Republic of China.

Received: 11 November 2017 Accepted: 1 August 2018 Published online: 10 August 2018

\section{References}

1. Koinis F, Kotsakis A, Georgoulias V, et al. Small cell lung cancer (SCLC): no treatment advances in recent years. Translational lung cancer research. 2016;5(1):39-50.

2. Sher T, Dy GK, Adjei AA, et al. Small cell lung cancer. Mayo Clin Proc. 2008; 83(3):355-67.

3. Kalemkerian GP, Gadgeel SM. Modern staging of small cell lung cancer. J Natl Compr Cancer Netw. 2013;11(1):99-104.
4. Micke P, Faldum A, Metz T, et al. Staging small cell lung cancer: veterans administration lung study group versus International Association for the Study of Lung Cancer--what limits limited disease? Lung Cancer. 2002;37(3):271-6.

5. Davies AM, Lara PN, Lau DH, Gandara DR. Treatment of extensive small cell lung cancer. Hematol Oncol Clin North Am. 2004;18(2):373-85.

6. Jiang J, Liang $X$, Zhou $X$, et al. A meta-analysis of randomized controlled trials comparing irinotecan/platinum with etoposide/platinum in patients with previously untreated extensive-stage small cell lung cancer. Journal of thoracic oncology: official publication of the International Association for the Study of Lung Cancer. 2010;5(6):867-73.

7. Tierney JF, Stewart LA, Ghersi D, et al. Practical methods for incorporating summary time-to-event data into meta-analysis. Trials. 2007;8:16.

8. Shi Y, Hu Y, Hu X, et al. Cisplatin combined with irinotecan or etoposide for untreated extensive-stage small cell lung cancer: a multicenter randomized controlled clinical trial. Thoracic cancer. 2015;6(6):785-91.

9. Schmittel A, Sebastian M, Fischer von Weikersthal L, et al. A German multicenter, randomized phase III trial comparing irinotecan-carboplatin with etoposide-carboplatin as first-line therapy for extensive-disease smallcell lung cancer. Annals of oncology: official journal of the European Society for Medical Oncology/ESMO. 2011;22(8):1798-804.

10. Zatloukal P, Cardenal F, Szczesna A, et al. A multicenter international randomized phase III study comparing cisplatin in combination with irinotecan or etoposide in previously untreated small-cell lung cancer patients with extensive disease. Annals of oncology : official journal of the European Society for Medical Oncology/ESMO. 2010;21(9):1810-6.

11. Lara PN Jr, Natale R, Crowley J, et al. Phase III trial of irinotecan/cisplatin compared with etoposide/cisplatin in extensive-stage small-cell lung cancer: clinical and pharmacogenomic results from SWOG S0124. J Clin Oncol Off J Am Soc Clin Oncol. 2009;27(15):2530-5.

12. Hermes A, Bergman B, Bremnes R, et al. Irinotecan plus carboplatin versus oral etoposide plus carboplatin in extensive small-cell lung cancer: a randomized phase III trial. J Clin Oncol Off J Am Soc Clin Oncol. 2008;26(26):4261-7.

13. Pan D, Hou M, Li H, et al. Irinotecan plus cisplatin compared with etoposide plus cisplatin for small cell lung cancer: a randomized clinical trial. Zhongguo fei ai za zhi = Chinese journal of lung cancer, 2006:9(5):443-6.

14. Hanna N, Bunn PA Jr, Langer C, et al. Randomized phase III trial comparing irinotecan/ cisplatin with etoposide/cisplatin in patients with previously untreated extensive-stage disease small-cell lung cancer. J Clin Oncol Off J Am Soc Clin Oncol. 2006;24(13):2038-43.

15. Noda K, Nishiwaki Y, Kawahara M, et al. Irinotecan plus cisplatin compared with etoposide plus cisplatin for extensive small-cell lung cancer. N Engl J Med. 2002:346(2):85-91.

16. Kim DW, Kim HG, Kim JH, Park K, Kim HK, Jang JS, et al. Randomized phase III trial of irinotecan plus cisplatin versus etoposide plus cisplatin in chemotherapy-Naïve Korean patients with extensive-disease small cell lung Cancer. Cancer Res Treat. 2018 Mar 12; https://doi.org/10.4143/crt.2018.019.

17. Higgins JPT, Green S. Cochrane Handbook for Systematic Reviews of Interventions Version 5.0.2 [updated September 2009]. The Cochrane Collaboration. 2008; Available from www.cochrane-handbook.org.

18. Johnson BE, Janne PA. Basic treatment considerations using chemotherapy for patients with small cell lung cancer. Hematol Oncol Clin North Am. 2004;18(2):309-22.

19. Ando Y, Chida M, Nakayama K, et al. The UGT1A1*28 allele is relatively rare in a Japanese population. Pharmacogenetics. 1998;8(4):357-60.

20. Liu CY, Chen PM, Chiou TJ, et al. UGT1A1*28 polymorphism predicts irinotecaninduced severe toxicities without affecting treatment outcome and survival in patients with metastatic colorectal carcinoma. Cancer. 2008:112(9):1932-40.

21. Lei Jiang K-HY, Deng-hai Mi, Ya-li Liu, et al. Safety of irinotecan/ cisplatin versus etoposide/ cisplatin for patients with extensive-stage small-cell lung Cancer: a Meta analysis. Clinical Lung Cancer, September 2007:8(8):497-501.

22. Gagne JF, Montminy V, Belanger P, Journault K, GaucherG, Guillemette C (2002) Common human UGT1A polymorphisms and the altered metabolism of irinotecan active metabolite 7-ethyl-10-hydroxycamptothecin (SN-38) Mol Pharmacol62(3):608-617.

23. Jinno H, Tanaka-Kagawa T, Hanioka N, Saeki M, Ishida S, Nishimura T, Ando M, Saito Y, Ozawa S, Sawada J. Glucuronidation of 7-ethyl-10-hydroxycamptothecin (SN-38), an active metabolite of irinotecan (CPT-11), by human UGT1A1 variants, G71R, P229Q, and Y486D. Drug Metab Dispos. 2003;31(1):108-13.

24. Cheng L, Li M, Hu J, et al. UGT1A1*6 polymorphisms are correlated with irinotecan-induced toxicity: a system review and meta-analysis in Asians. Cancer Chemother Pharmacol. 2014;73(3):551-60. 